\title{
Componentes morfogenéticos y acumulación del pasto mulato a diferente frecuencia e intensidad de pastoreo
}

\section{Morphogenetic components and accumulation in mulato grass at different frequencies and intensities of grazing}

\author{
Aldenamar Cruz-Hernández ${ }^{\text {** }}$, Alfonso Hernández-Garaya , Humberto Vaquera-Huertab, Alfonso \\ Chay-Canula , J avier Enríquez-Quiroz ${ }^{c}$, Santiago Ramírez-Vera ${ }^{a}$
}

\begin{abstract}
RESUMEN
En las regiones tropicales las gramíneas representan la principal fuente de alimentación para los rumiantes, por lo que el rendimiento y la calidad forrajera son de gran interés para mitigar la baja productividad. La mayoría de los estudios en forrajes se basan en la producción de la materia seca y el valor nutritivo, sin considerar los caracteres morfogenéticos y estructurales del pasto, en la dinámica de rebrote. En Brachiaria hibrido cv mulato se estudiaron a 14, 21 y 28 días de pastoreo, y 9-11, 13-15 cm de altura residual, que se distribuyeron en un diseño de bloques al azar en arreglo factorial 3 x 2 con tres repeticiones. Se midió: acumulación de forraje, densidad poblacional de tallos, tasa de aparición y muerte de tallos, recambio de tejido foliar. La mayor acumulación del forraje fue de 2,769, 4,985, 7,033 kg de materia seca por hectárea con pastoreo ligero en lluvias. La tasa de aparición de tallos con el pastoreo severo superó en 13, 8 y $14 \%$ al ligero en las épocas de nortes, seca y lluvias en el primer ciclo. La mayor elongación foliar se obtuvo al pastorear cada 28 días en la época de lluvias. Se concluye que durante la época de lluvias el pasto mulato presentó la mayor acumulación del forraje al cosecharse en periodos de 28 días con pastoreo ligero. La tasa de elongación y senescencia foliar mostraron los mayores valores al pastorear ligeramente la pradera cada 28 días con una densidad menor de tallos.
\end{abstract}

PALABRAS CLAVE: Forraje, Brachiaria hibrido, Dinámica de tallos, Tejido foliar.

\begin{abstract}
Grasses are the main feed source for ruminant livestock in tropical regions. Forage yield and quality are therefore extremely important for mitigating low productivity. Most studies of forage are based on dry matter production and nutritional value, and ignore grass morphogenetic and structural traits in the resprouting dynamic. Brachiaria hibrido cv. mulato was studied at 14, 21 and $28 \mathrm{~d}$ grazing frequencies, and 9-11 and 13-15 cm residual heights using a random block design and a $3 \times 2$ factorial arrangement with three replicates. Five variables were measured: forage accumulation; population stem density; stem appearance rate; stem death rate; and leaf tissue turnover. At all three frequencies, forage accumulation was highest under light grazing during the rainy season (2,769 $\mathrm{kg}$ dry matter at $14 \mathrm{~d} ; 4,985 \mathrm{~kg}$ at $21 \mathrm{~d}$; and 7,033 $\mathrm{kg}$ at $28 \mathrm{~d})$. Under severe grazing in the first cycle, stem appearance rate was higher than under light grazing by $13 \%$ in the northwinds season, by $8 \%$ in the dry season and by $14 \%$ in the rainy season. Leaf elongation was greatest at the $28 \mathrm{~d}$ frequency during the rainy season. Mulato grass exhibited the highest forage accumulation during the rainy season when harvested at a 28 d frequency under light grazing. Leaf elongation rate and leaf senescence exhibited the highest values when grazed lightly every $\mathbf{2 8} \mathbf{d}$ at a low stem density.
\end{abstract}

KEY WORDS: Forage, Brachiaria hibrido, Stem dynamic, Leaf tissue.

Recibido el 15 de diciembre de 2015. Aceptado el 28 de enero de 2016.

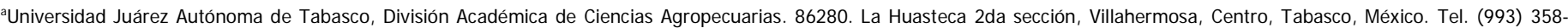
1585,142-9151, Fax (993) 142-9150.

bPosgrado de Ganadería, Colegio de Postgraduados Montecillo, Estado de México. México.

'Campo Experimental Cotaxtla. Instituto Nacional de Investigaciones Forestales Agrícolas y Pecuarias. Isla, Veracruz, México.

*autor de correspondencia (ingaldecruz@gmail.com). 
En las regiones tropicales la principal fuente de alimentación para los rumiantes son las gramíneas, por lo que el rendimiento y la calidad forrajera son de gran interés para mitigar la baja productividad en los sistemas de pastoreo. Sin embargo, en estas regiones la producción del forraje y su uso se determina por las necesidades de alimentar a los rumiantes, sin considerar el momento oportuno de cosecha para su utilización ${ }^{(1)}$. La mayoría de los estudios en forrajes utilizan la producción de materia seca (MS) y el valor nutritivo, sin considerar que los caracteres morfogenéticos y estructurales de las plantas son importante en la dinámica de rebrote y persistencia de las especies forrajeras ${ }^{(2)}$. En este sentido el manejo de las praderas se debe considerar estratégico; esto implica conocer los principales componentes que afectan al rendimiento y la calidad de las praderas, dentro de los que se encuentran la frecuencia y la altura residual; los cambios que ocurran en estos componentes propiciarían modificaciones e incluso la desaparición de las especies deseadas ${ }^{(3)}$. Estudios con diferentes especies forrajeras tropicales, indican que para utilizar eficientemente la pradera, la altura propicia del forraje residual del pasto Panicum maximum J acq cv.Tanzania es de 25 a $50 \mathrm{~cm}$ con el $95 \%$ de intercepción luminosa ${ }^{(4,5)}$ y de 15 a $30 \mathrm{~cm}$ para Brachiaria brizantha cv. Marandu con tres y cuatro hojas por tallo(6). Estos autores consideran que a menores alturas residuales se afecta al rendimiento de la pradera y la respuesta animal. Sin embargo, se ha demostrado que al cosechar a $10 \mathrm{~cm}$ de altura residual, el pasto Brachiaria brizantha cv. Marandu aumenta la velocidad del flujo de tejido e incrementa la tasa de aparición y muerte de tallos ${ }^{(7)}$.

En México existen pocos estudios sobre caracteres morfogenéticos y estructurales de pastos tropicales; estos datos permiten enfatizar la importancia que representan en el rendimiento de las especies forrajeras. La acumulación de los forrajes también se le puede atribuir al incremento en la densidad de tallos, en el peso individual de cada tallo o a una combinación de $\operatorname{ambos}^{(8,9)}$, esto se debe a que el tallo se considera como la unidad de crecimiento primario y la pradera puede considerarse como una población de estos. Un estudio con pasto capim-marandu muestra mayor población de tallos al cosecharse a $15 \mathrm{~cm}$ de altura residual, que a $30 \mathrm{~cm}$, con intervalo de defoliación de tres hojas por tallo(6).

El pasto mulato, es una especie forrajera perenne con potencial productivo entre 10 a $25 \mathrm{t}$ de materia seca (MS) ha-1 año $^{-1}$, proteína cruda de 90 a $170 \mathrm{~g} \mathrm{~kg}^{-1}$ MS y 550 a $620 \mathrm{~g} \mathrm{~kg}^{-1}$ de digestibilidad in vitro de la $\mathrm{MS}^{(10)}$. Sin embargo, la mayoría de las pocas investigaciones que se han hecho se enfocan a producción de MS y valor nutritivo, sin considerar el papel importante de los caracteres morfogenéticos y estructurales en la dinámica de rebrote y persistencia de las gramíneas. El objetivo de esta investigación fue determinar el efecto de las diferentes frecuencias e intensidades de pastoreo, en la producción de la materia seca, dinámica poblacional de tallos y recambio de tejido foliar del pasto mulato.

El estudio se realizó de noviembre 2007 a mayo 2009 en el área experimental de la División Académica de Ciencias Agropecuarias de la Universidad Juárez Autónoma de Tabasco (UJ AT), que se localiza a 170 46’ 56" N y 920 57' $28^{\prime \prime} \mathrm{O}$, a 10 msnm y se ubica en el km 25 de la carretera Villahermosa - Teapa, en el municipio del Centro, Tabasco, México. El sitio tiene precipitación y temperatura promedio anual de $2,010 \mathrm{~mm}$ y $27.2^{\circ} \mathrm{C}$ y corresponde a un clima $A f^{(11)}$ y suelo luvisol crómico ${ }^{(12)}$. Los datos de precipitación y temperatura máxima y mínima durante el periodo experimental, se obtuvieron con instrumentales meteorológica de la UJ AT (Figura 1).

La siembra del pasto se realizó por semillas y manualmente a piquete, en julio de 2006. Antes de sembrar se aplicó $1 \mathrm{~L} \mathrm{ha}^{-1}$ de herbicida glifosato al $41 \%$ para eliminar las malezas presentes. Para el establecimiento de la pradera, se utilizaron $6 \mathrm{~kg}$ de semillas ha ${ }^{-1}$, con una distribución de $50 \mathrm{~cm}$ entre plantas; posteriormente se seleccionó un área de $1,800 \mathrm{~m}^{2}$ (37.5 x $48 \mathrm{~m}$ ) que se dividió en 18 unidades experimentales, con una superficie por unidad de $100 \mathrm{~m}^{2}$ (12.5 x $\left.8 \mathrm{~m}\right)$.

Los tratamientos se distribuyeron aleatoriamente en un diseño de bloques al azar con tres repeticiones y un arreglo factorial $3 \times 2$, donde los factores fueron tres frecuencias de pastoreos (FP: 14, 21, 28 días) y 
dos intensidades o alturas residuales de pastoreo (severo 9-11 y ligero 13-15 cm altura). Quince días antes del inicio del estudio, las praderas presentaban entre 35 a $45 \mathrm{~cm}$ de altura, por lo que, se realizó un pastoreo de uniformización para lo cual se utilizaron 10 becerros de 180 a $230 \mathrm{~kg}$ de peso vivo, los animales permanecieron en todas las unidades experimentales hasta alcanzar la altura residual correspondiente. No se utilizó fertilizante antes ni durante el experimento.

Para evaluar la acumulación del forraje, un día antes de iniciar el estudio, se colocaron aleatoriamente, en cada unidad experimental, dos cuadros fijos de $0.5 \mathrm{~m}^{2}$, en los cuales se cosechó todo el forraje presente un día antes de cada pastoreo de acuerdo a los tratamientos; posteriormente el forraje se depositó en bolsas de papel debidamente etiquetadas, se pesaron y se colocaron dentro de una estufa de aire forzado a 60 oC durante $72 \mathrm{~h}$, después se retiraron, se pesaron en una balanza digital marca Scout ${ }^{\circledR}$ Pro y por diferencia de peso se obtuvo la MS. Posteriormente, se utilizaron cinco becerros de 180 a $230 \mathrm{~kg}$ de peso vivo únicamente como cosechadores por unidad experimental, hasta alcanzar la intensidad del pastoreo correspondiente. Se evaluaron dos ciclos, el primero fue de noviembre 2007 a octubre 2008, y el segundo ciclo de noviembre 2008 a mayo 2009. En ambos ciclos se presentaron las épocas de los nortes (noviembre-febrero) y seca (marzo-abril), la época de lluvias (junio-octubre) solo se consideró en el primer ciclo. Para la región tropical húmeda la presencia de precipitación y temperatura son bien marcadas, por lo que, en el presente experimento la acumulación de forraje para su análisis se agrupó por épocas.

Para la dinámica poblacional de tallos, en cada unidad experimental se colocaron dos aros de $20 \mathrm{~cm}$ de diámetro a nivel de suelo y se dejaron fijos durante todo el periodo experimental, y todos los tallos dentro del aro se marcaron con anillos de alambre de un mismo color, que se consideraron como la población inicial; los tallos vivos y muertos se registraron a intervalos de 30 días y los tallos nuevos se marcaron con un nuevo color, cada color representó una generación de tallos. Los datos individuales en cada aro, se utilizaron para calcular los cambios en la dinámica de tallos, tasa de aparición y tasa de muerte de tallos; posteriormente se calcularon tallos por metro cuadrado, para su posterior análisis, con la suposición de que toda la

Figura 1. Datos mensuales de precipitación y temperaturas, del sitio experimental




superficie del metro, estaría ocupado con la misma densidad que la obtenida en la muestra ${ }^{(13)}$.

La tasa de recambio de tejido foliar, se realizó a mediados de cada época, un día después del pastoreo; en cada unidad experimental se seleccionaron de manera aleatoria 10 tallos, los cuales se identificaron con anillos de plástico de color, y con una regla graduada en milímetros se midió en cada hoja, de cada tallo, la longitud de la lámina foliar, desde la lígula hasta el ápice en hojas verdes o hasta la base del tejido clorótico en hojas senescentes ${ }^{(2)}$. Posteriormente esta misma determinación se realizó cada semana hasta un día antes del siguiente pastoreo. La tasa de elongación foliar (TEF; $\mathrm{cm}$ tallo $\mathrm{o}^{-1} \mathrm{dí}^{-1}$ ), se calculó para las hojas en expansión, por la diferencias entre la longitud de las láminas foliares final (LLFf) menos la inicial (LLFi), al final de dos mediciones sucesivas divididas entre el número de días $(\mathrm{T})$ transcurridas entre ambas mediciones sucesivas con la siguiente fórmula TEF $=($ LLFf-LLFi)/T. La tasa de senescencia foliar (TSF; cm tallo día ${ }^{-1}$ ), se obtuvo para hojas maduras y en proceso de senescencia, como la diferencia entre la longitud de las láminas foliares verdes al inicio (LFVi) y al final de dos mediciones sucesivas (LFVf), dividida entre el número de días (T) que transcurrieron entre ambas mediciones. $\mathrm{TSF}=(\mathrm{LFVi}-\mathrm{LFV}) / \mathrm{T}$. La tasa de crecimiento neto foliar por tallo (CNF; cm tallo-1 día ${ }^{-1}$ ), se calculó como la diferencia entre la tasa de elongación foliar (TEF) y la tasa de senescencia foliar (TSF), CNF=TEF-TSF. Los datos se analizaron con el modelo PROC MIXED ${ }^{(14)}$. Los efectos de frecuencias, intensidades de pastoreos, épocas del año y sus interacciones, se consideraron fijos y el efecto de bloques se consideró aleatorio. La comparación de medias se realizó con la prueba de Tukey, $\mathrm{a}=0.05^{(15)}$.

Figura 2. Acumulación del pasto mulato a diferentes frecuencias (14, 21 y 28 días) e intensidades de pastoreo ( $A=9-11 \mathrm{~cm}$ y $B=13-15 \mathrm{~cm}$ de altura), $C 1=$ seca, nortes, lluvias. $C 2=$ seca y nortes

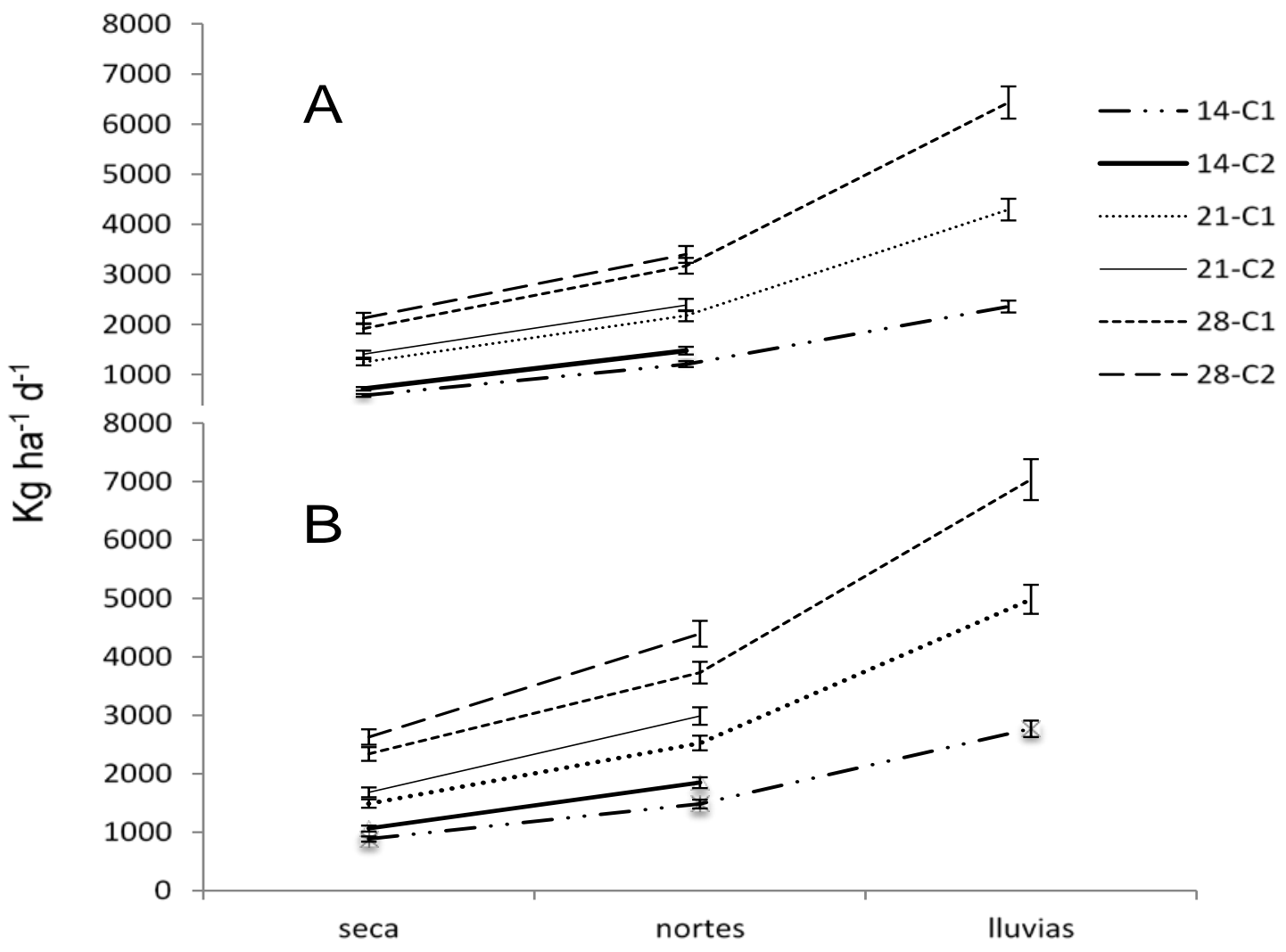

Épocas del año 
Las precipitaciones durante la fase experimental presentaron cambios diferenciales, con menor precipitación ( $262 \mathrm{~mm}$ ) en nortes del primer ciclo, y un aumento del $77 \%$ en el segundo ciclo (nortes); las mayores precipitaciones fueron 270, 545 y $296 \mathrm{~mm}$, que se presentaron en los meses de junio, septiembre y octubre (época de lluvias); contrariamente ocurrió en la época seca en ambos ciclos (Figura 1); sin embargo, en estas épocas se presentó la mayor temperatura promedio con $30 \stackrel{\circ}{\circ}$

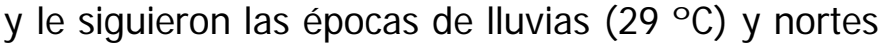
( $25 \stackrel{\circ}{ } \mathrm{C}$ ), en ambos ciclos. El pasto mulato tiene un rango amplio de adaptación y crece bien desde el nivel del mar hasta $1,800 \mathrm{~m}$ en condiciones de trópico húmedo con altas precipitaciones, y en condiciones subhúmedas con 5 a 6 meses secos y precipitación anual mayor a $700 \mathrm{~mm}^{(16)}$. En este sentido, la distribución de la precipitación pluvial, generó una condición creciente de deficiencia hídrica, durante la sequía, y buenas condiciones de humedad en el suelo, durante las Iluvias; como consecuencia, en estas épocas se propició la mayor acumulación de forraje (Figura 2).

Las frecuencias del pastoreo afectaron a la acumulación del forraje en todos los ciclos, con mayor acumulación en la época de lluvias en el primer ciclo $(P<0.05)$. Al aumentar el intervalo entre pastoreo de 14, 21 y 28 días, la producción de forraje se incrementó de 2,356 a 4,293 y 6,433 kg MS ha-1, vs 2,769, 4,985, 7,033 kg MS ha-1 con pastoreo severo vs ligero en la época de lluvias (Figura 2).

La respuesta de la pradera estuvo influenciada por el manejo; la menor acumulación de forrajes se obtuvo con pastoreo severo cada 14 días, ya que las plantas expuestas a defoliaciones severas, presentan disminución progresiva en la biomasa de hojas verdes conforme crece la pradera y desarrollan cambios en su morfología que les permite mantener área foliar verde por debajo de la altura de cosecha(17), esto explica la presencia de una baja tasa de elongación foliar cuando la pradera se pastoreaba cada 14 y 21 días. Estos cambios en la producción de biomasa posiblemente se debieron a los cambios en la densidad de tallos (Cuadro 1), ya que un aumento en la masa de forraje puede ocurrir por una reducción en la densidad poblacional de tallo, según la intensidad de pastoreo a la que se somete la pradera ${ }^{(18)}$, como ocurrió en el presente estudio, que al pastorear ligeramente cada 28 días, se presentó la menor densidad poblacional de tallos, pero la acumulación de forraje fue mayor. Contrariamente ocurrió al cosechar a 14 días. Estudios recientes consignan que al pastorear intensamente la pradera es mayor la eliminación de los meristemos apicales ${ }^{(19)}$.

Respecto a la dinámica de tallos, la frecuencia de pastoreo afectó la densidad de tallos (DT) y la tasa de aparición de tallos (TAT) $(P<0.05)$ en ambos

Cuadro 1. Cambios estacionales en la densidad de tallos del pasto mulato (Brachiaria hibrido, 36061) a diferentes frecuencias e intensidades de pastoreo

\begin{tabular}{|c|c|c|c|c|c|c|}
\hline \multicolumn{2}{|c|}{ Tratamientos } & \multicolumn{3}{|c|}{ Ciclo 1} & \multicolumn{2}{|c|}{ Ciclo 2} \\
\hline \multirow[t]{2}{*}{$\begin{array}{l}\text { Frecuencia } \\
\text { (días) }\end{array}$} & $\begin{array}{l}\text { Intensidad } \\
(\mathrm{cm})\end{array}$ & Nortes & Seca & Lluvias & \multirow[t]{2}{*}{ Nortes } & \multirow[t]{2}{*}{ Seca } \\
\hline & & \multicolumn{3}{|c|}{ Densidad de tallos (tallos m-2) } & & \\
\hline 14 & & $3635 \mathrm{Da}$ & $4843 \mathrm{Ca}$ & $5644 \mathrm{Ba}$ & 6559 Аа & 5866 Aba \\
\hline 21 & & $2955 \mathrm{Db}$ & $3684 \mathrm{cb}$ & $4351 \mathrm{ABb}$ & $4869 \mathrm{Ab}$ & $4140 \mathrm{BCb}$ \\
\hline \multirow[t]{3}{*}{28} & & $2608 \mathrm{Db}$ & $3324 \mathrm{CDb}$ & $4168 \mathrm{ABb}$ & $4394 \mathrm{Ab}$ & $3489 \mathrm{BCC}$ \\
\hline & Severo & $3280 \mathrm{Da}$ & $4222 \mathrm{Ca}$ & 5079 ABa & 5729 Аа & $4979 \mathrm{Ba}$ \\
\hline & Ligero & $2857 \mathrm{Db}$ & $3679 \mathrm{cb}$ & $4363 \mathrm{ABb}$ & $4818 \mathrm{Ab}$ & $4018 \mathrm{BCb}$ \\
\hline EEM & & 58.8 & 104.6 & 96.1 & 86.0 & 91.7 \\
\hline \multicolumn{2}{|c|}{ Frecuencia de pastoreo } & ** & ** & ** & ** & ** \\
\hline \multicolumn{2}{|c|}{ Intensidad de pastoreo } & * & * & ** & ** & ** \\
\hline
\end{tabular}

abc= Diferente literal minúscula, en cada columna, indican diferencia $(P<0.05) ; A B C D=$ Diferente literal mayúscula, en cada hilera, indican diferencia $(P<0.05)$. EEM= Error estándar de la media. ${ }^{\star} P \leq 0.01$ : ${ }^{*} P \leq 0.05$. 
ciclos solo se registró efecto de intensidad para la TAT $(P<0.05)$ en las épocas de nortes y seca en el ciclo 1. La TAT en la época de lluvias disminuyó en 17 y $40 \%$ (ciclo 1 ), en tanto que para las épocas de nortes decreció en 29 y $63 \%$ en el primer ciclo y 18 y $37 \%$ en el segundo ciclo, al ampliar el intervalo entre pastoreos de 14 a 21 y 28 días, respectivamente (Cuadro 2).

En general, la TAT con el pastoreo severo superó en 13, 8 y $14 \%$ al ligero en las épocas de nortes, seca y lluvias durante el ciclo $1(P<0.05)$. Durante la época de nortes del ciclo 2, la TAT fue $9 \%$ $(P<0.05)$ mayor con el pastoreo severo en comparación con el ligero. Con respecto a la tasa de mortalidad de tallos (TMT), solo se registró efecto de la frecuencia de pastoreo en la época de lluvias $(P<0.05)$, este efecto propició que la TMT disminuyera en un 10 y $30 \%$ al ampliar el intervalo entre pastoreo de 14 a 21 y 28 días, respectivamente. Los resultados anteriores están fuertemente influenciados, en su mayoría, por factores de manejo, principalmente por la frecuencia e intensidad de pastoreo a la que se sometió la pradera. Estos factores contribuyeron a una mayor TAT en las épocas de lluvias y nortes en ambos ciclos. Así mismo, la alta precipitación en la época de lluvias permitió mantener mayor humedad en el suelo, lo que propició condiciones favorables para la generación de nuevos rebrotes en la época de nortes del ciclo 2, en tanto que, la menor TAT en la época de seca en ambos ciclos, se debió a falta de humedad, ya que la temperatura durante el experimento estuvo dentro de los 30 y $34{ }^{\circ} \mathrm{C}^{(20)}$, temperatura óptima para forrajes tropicales.

La mayor densidad de tallos se registró al pastorear cada 14 días, y fue consecuencia de la mayor TAT, que concuerda con la mayor TMT (Cuadro 3). La mayor tasa de muerte de tallos se asocia con una mayor tasa de aparición de tallos ${ }^{(6)}$, asociación que en este estudio se observó en las épocas de nortes y lluvias en ambos ciclos. Sin embargo, la disponibilidad de nutrientes y las condiciones ambientales como humedad, luz y temperatura fueron los principales factores que propiciaron una alta $\operatorname{TMT}^{(7,17)}$.

Cuadro 2. Cambios estacionales en la tasa de aparición y muerte de tallos del pasto mulato (Brachiaria hibrido, 36061) a diferentes frecuencias e intensidades de pastoreo

\begin{tabular}{|c|c|c|c|c|c|c|}
\hline \multicolumn{2}{|c|}{ Tratamientos } & \multicolumn{3}{|c|}{ Ciclo 1} & \multicolumn{2}{|c|}{ Ciclo 2} \\
\hline $\begin{array}{l}\text { Frecuencia } \\
\text { (días) }\end{array}$ & $\begin{array}{l}\text { Intensidad } \\
\text { (cm) }\end{array}$ & Nortes & Seca & Lluvias & Nortes & Seca \\
\hline & & \multicolumn{5}{|c|}{ Tasa de aparición de tallos (tallos $\mathrm{m}^{-2} \mathrm{~d}^{-1}$ ) } \\
\hline 14 & & $31 \mathrm{Aa}$ & $15 \mathrm{Ba}$ & $28 \mathrm{Aa}$ & $26 \mathrm{Aa}$ & $12^{\mathrm{Bb}}$ \\
\hline 21 & & $24 \mathrm{Aab}$ & $12 \mathrm{Bb}$ & 24 Aab & 22 Aab & $11 \mathrm{Ba}$ \\
\hline \multirow[t]{3}{*}{28} & & $19 \mathrm{Aa}$ & $10^{\mathrm{Bb}}$ & $20 \mathrm{Ab}$ & $19 \mathrm{Ab}$ & 8 Ba \\
\hline & Severo & $27 \mathrm{Aa}$ & $13 \mathrm{Ba}$ & $25 \mathrm{Aa}$ & $23 \mathrm{Aa}$ & $10 \mathrm{Ba}$ \\
\hline & Ligero & $22 \mathrm{Aa}$ & $12 \mathrm{Ba}$ & $22 \mathrm{Aa}$ & $21 \mathrm{Aa}$ & $10 \mathrm{Ba}$ \\
\hline EEM & & 1.2 & 0.3 & 0.6 & 0.8 & 0.2 \\
\hline Frecuencia o & toreo & * & ** & ** & ** & ** \\
\hline \multirow{2}{*}{\multicolumn{2}{|c|}{ Intensidad de pastoreo }} & * & * & ns & ns & ns \\
\hline & & \multicolumn{5}{|c|}{ Tasa de mortalidad de tallos (tallos $\mathrm{m}^{-2} \mathrm{~d}^{-1}$ ) } \\
\hline 14 & & $11 \mathrm{Ba}$ & $9 \mathrm{Ba}$ & $20 \mathrm{Aa}$ & $23 \mathrm{Aa}$ & $24 \mathrm{Aa}$ \\
\hline 21 & & $10 \mathrm{Ba}$ & $7 \mathrm{Ba}$ & $18 \mathrm{Aa}$ & $20 \mathrm{Aa}$ & $22 \mathrm{Aa}$ \\
\hline \multirow[t]{3}{*}{28} & & $6 \mathrm{Ca}$ & $6 \mathrm{Ca}$ & $12 \mathrm{Bb}$ & $22 \mathrm{Aa}$ & $20 \mathrm{Aa}$ \\
\hline & Severo & $9 \mathrm{Ca}$ & $8 \mathrm{Ca}$ & $17 \mathrm{Ba}$ & $23 \mathrm{Aa}$ & $22 \mathrm{ABa}$ \\
\hline & Ligero & $8 \mathrm{Ca}$ & $7 \mathrm{Ca}$ & $16 \mathrm{Ba}$ & $21 \mathrm{ABa}$ & $21 \mathrm{Aa}$ \\
\hline EEM & & 0.7 & 0.5 & 0.5 & 1.0 & 0.7 \\
\hline \multirow{2}{*}{\multicolumn{2}{|c|}{$\begin{array}{l}\text { Frecuencia de pastoreo } \\
\text { Intensidad de pastoreo }\end{array}$}} & ns & ns & ** & ns & ns \\
\hline & & ns & ns & ns & ns & ns \\
\hline
\end{tabular}

$a b c=$ Diferente literal minúscula, en cada columna, indican diferencia $(P<0.05) ; A B C D=$ Diferente literal mayúscula, en cada hilera, indican diferencia $(P<0.05)$. EEM= Error estándar de la media. ns= no significativo ${ }^{* *} P \leq 0.01 ;{ }^{*} P \leq 0.05$; 
A pesar de no haber efecto significativo entre tratamientos en las épocas de los nortes en ambos ciclos, la menor TMT se observó al pastorear cada 28 días. Probablemente la planta interceptó mayor porcentaje de luz solar por la alta concentración de tejido foliar (Figura 2), que propició un aumento en sus reservas de carbohidratos, y le permitió a la planta evitar mayor muerte de tallos; sin embargo, al disminuir la cantidad y calidad de luz que llega hacia los estratos inferiores, se inhibió la aparición de nuevos tallos, por falta de estímulos para la activación de los meristemos de crecimiento ${ }^{(1,9)}$. Tal como lo consignan algunos autores que al incrementar la altura residual de 10 a $40 \mathrm{~cm}$ y de 30 a $50 \mathrm{~cm}$ en los pastos Brachiaria brizantha cv. Marandu y Panicum maximum Jacq. cv. Tanzania encontraron menor tasa de aparición de tallos ${ }^{(7,19)}$.

Se registró un efecto de frecuencia de pastoreo en la tasa de elongación foliar $(P<0.05)$ durante todo el periodo de evaluación, y un efecto de intensidad de pastoreo en la época de nortes del ciclo 2 (Cuadro 3). La elongación foliar se incrementó al aumentar el intervalo entre pastoreos en todas las épocas. Durante las lluvias la elongación foliar al pastorear cada 28 días superó en 25 y $159 \%$ al de 21 y 14 días, respectivamente. En la época de nortes del ciclo 1 al pastorear cada 28 días se registró una mayor elongación de 213 y $216 \%$, vs 3 y $167 \%$ (en época de nortes) que el pastoreo a 21 y 14 días. Durante la época de seca del primer ciclo las frecuencias de corte de 14 y 21 días tuvieron 159 y $25 \%$, 230 y $41 \%$ (época de seca; ciclo 2 ) menor tasa de elongación que el de 28 días.

La tasa de elongación con el pastoreo ligero es mayor a la del severo y únicamente diferente en la época de seca en el ciclo $2(P<0.05)$, con $45 \%$ mayor elongación que con el severo. La tasa de senescencia foliar presentó la misma tendencia que la tasa de elongación foliar. Independientemente del ciclo, la senescencia foliar aumentó conforme el

Cuadro 3. Cambios estacionales en la tasa de elongación, senescencia y crecimiento neto foliar del pasto mulato (Brachiaria hibrido, 36061) a diferentes frecuencias e intensidades de pastoreo

\begin{tabular}{|c|c|c|c|c|c|c|}
\hline \multicolumn{2}{|c|}{ Tratamientos } & \multicolumn{3}{|c|}{ Ciclo 1} & \multicolumn{2}{|c|}{ Ciclo 2} \\
\hline $\begin{array}{l}\text { Frecuencia } \\
\text { (días) }\end{array}$ & $\begin{array}{l}\text { Intensidad } \\
(\mathrm{cm})\end{array}$ & Nortes & Seca & Lluvias & Nortes & Seca \\
\hline & & \multicolumn{5}{|c|}{ Tasa de elongación foliar (cm tallo-1 día-1) } \\
\hline \multicolumn{2}{|l|}{14} & $1.03 \mathrm{Ab}$ & $0.74 \mathrm{Bc}$ & $1.86 \mathrm{Ac}$ & $1.06 \mathrm{Bb}$ & $0.76^{\mathrm{Bc}}$ \\
\hline \multicolumn{2}{|l|}{21} & $1.04 \mathrm{Cb}$ & $1.80 \mathrm{cb}$ & $3.87 \mathrm{Ab}$ & $2.76 \mathrm{Ba}$ & $1.78 \mathrm{cb}$ \\
\hline \multicolumn{2}{|l|}{28} & $3.26 \mathrm{Ba}$ & $2.71 \mathrm{Ba}$ & $4.82 \mathrm{Aa}$ & $2.83 \mathrm{Ba}$ & $2.51 \mathrm{Ba}$ \\
\hline & Severo & $1.68 \mathrm{Ba}$ & $1.53 \mathrm{Ba}$ & $3.37 \mathrm{Aa}$ & $2.15 \mathrm{Ba}$ & $1.37 \mathrm{Bb}$ \\
\hline & Ligero & $1.87 \mathrm{Ba}$ & $1.97 \mathrm{Ba}$ & $3.66 \mathrm{Aa}$ & $2.29 \mathrm{Ba}$ & $2.00 \mathrm{Ba}$ \\
\hline \multirow{4}{*}{\multicolumn{2}{|c|}{$\begin{array}{l}\text { EEM } \\
\text { Frecuencia de pastoreo } \\
\text { Intensidad de pastoreo }\end{array}$}} & 0.17 & 0.31 & 0.30 & 0.27 & 0.13 \\
\hline & & ** & ** & ** & ** & ** \\
\hline & & ns & ns & ns & ns & * \\
\hline & & \multicolumn{5}{|c|}{ Tasa de senescencia foliar (cm tallo-1 día-1) } \\
\hline \multicolumn{2}{|l|}{14} & $0.00 \mathrm{Ab}$ & $0.13 \mathrm{Aa}$ & $0.01 \mathrm{Ab}$ & $0.05^{\mathrm{Ab}}$ & $0.01 \mathrm{Aa}$ \\
\hline \multicolumn{2}{|l|}{21} & $0.09 \mathrm{Ab}$ & $0.11 \mathrm{Aa}$ & $0.11 \mathrm{Aab}$ & $0.10 \mathrm{Ab}$ & 0.33 Aa \\
\hline \multirow[t]{3}{*}{28} & & $0.62 \mathrm{Aa}$ & $0.41 \mathrm{ABa}$ & $0.35 \mathrm{ABa}$ & $0.30 \mathrm{Ba}$ & $0.30 \mathrm{Ba}$ \\
\hline & Severo & $0.21 \mathrm{Aa}$ & $0.21 \mathrm{Aa}$ & $0.14 \mathrm{Aa}$ & $0.15 \mathrm{Aa}$ & $0.26 \mathrm{Aa}$ \\
\hline & Ligero & $0.27 \mathrm{Aa}$ & $0.23 \mathrm{Aa}$ & $0.17 \mathrm{Aa}$ & $0.15 \mathrm{Aa}$ & $0.17 \mathrm{Aa}$ \\
\hline \multirow{3}{*}{\multicolumn{2}{|c|}{$\begin{array}{l}\text { EEM } \\
\text { Frecuencia de pastoreo } \\
\text { Intensidad de pastoreo }\end{array}$}} & 0.48 & 0.13 & 0.08 & 0.04 & 0.12 \\
\hline & & ** & ** & ** & * & * \\
\hline & & ns & ns & ns & ns & ns \\
\hline
\end{tabular}

$a b=$ Diferente literal minúscula, en cada columna, indican diferencia $(P<0.05) ; A B C D=$ Diferente literal mayúscula, en cada hilera, indican diferencia $(P<0.05)$. EEM= Error estándar de la media. ns= no sianificativo: ${ }^{* \star} P \leq 0.01:{ }^{*} P \leq 0.05$. 
intervalo de pastoreo se incrementó de 14 a 28 días $(P<0.05)$. Al pastorear ligeramente a los 28 días se registró mayor senescencia foliar que con el pastoreo severo $(P<0.05)$.

Respecto al flujo de tejido foliar, los resultados muestran que la velocidad de crecimiento del pasto varió entre épocas de acuerdo con las condiciones ambientales, y dentro de cada época con base en el manejo del pastoreo, como se ha demostrado en otros estudios en praderas cultivadas con pasto Mombaza y $B$. humidicola, respectivamente ${ }^{(2,8)}$.

En general, se observó un aumento progresivo en la tasa de elongación de las hojas al aumentar la altura residual y pastorear las praderas menos frecuentemente, propiciando los mayores valores de elongación y senescencia foliar (Cuadro 4). Tal como lo demuestra Difante ${ }^{(6)}$, al ampliar el intervalo de la cosecha, la elongación de hojas es mayor, contrariamente observaron que al pastorear severamente hay remoción de meristemos apicales, lo que puede propiciar la desaparición del pasto mulato.

La frecuencia de pastoreo afectó la tasa de crecimiento neto foliar (TCNF) en todos los ciclos $(P<0.01)$ y las intensidades de pastoreos solamente afectaron a la época de seca del ciclo $2(P<0.01)$. La TCNF se incrementó al aumentar el intervalo entre pastoreos en todos los ciclos (Cuadro 4). Durante la época de lluvias la TCNF aumentó en 103.8 y $143.4 \%$ al incrementar el intervalo de pastoreo de 14 a 21 y 28 días, respectivamente, respuestas similares se obtuvieron en las épocas de los nortes y seca en ambos ciclos. La TCNF con el pastoreo ligero en las épocas de nortes, seca y lluvias en el primer ciclo, se incrementó en un $9.5,19$ y $8.3 \%$ que con el pastoreo severo. En el segundo ciclo la TCNF aumentó 7 y $65 \%$ en las épocas de nortes y seca. Esto hace evidente que al pastorear intensamente la pradera, la cantidad de hoja removida por el animal propicia lenta recuperación de la planta, por lo que requiere de mayor tiempo para que se utilice nuevamente la pradera, como se presentó en el presente estudio al ampliar el intervalo de pastoreo. Estudios con otras especies forrajeras demuestran que al incrementar el intervalo de corte de 3 a 7 semanas, la tasa de crecimiento y senescencia foliar se incrementan propiciando menor crecimiento neto foliar en $P$. máximum cv. Mombaza(2). Similares resultados obtuvieron otros autores con el pasto Brachiaria brizantha cv. Marandu al aumentar la altura residual de 15 a $30 \mathrm{~cm}$ con tres y cuatro hojas por tallo ${ }^{(6)}$.

Se concluye que durante la época de lluvias el pasto mulato presentó mayor acumulación del forraje al cosecharse en periodos de 28 días con pastoreos ligeros. De acuerdo a las condiciones ambientales de cada época, la tasa de elongación y

Cuadro 4. Cambios estacionales en la tasa de crecimiento neto foliar del pasto mulato (Brachiaria hibrido, 36061) a diferentes frecuencias e intensidades de pastoreo

\begin{tabular}{|c|c|c|c|c|c|c|}
\hline \multicolumn{2}{|c|}{ Tratamientos } & \multicolumn{3}{|c|}{ Ciclo 1} & \multicolumn{2}{|c|}{ Ciclo 2} \\
\hline $\begin{array}{c}\text { Frecuencia } \\
\text { (días) }\end{array}$ & $\begin{array}{l}\text { Intensidad } \\
\text { (cm) }\end{array}$ & Nortes & Seca & Lluvias & Nortes & Seca \\
\hline & & \multicolumn{5}{|c|}{ Tasa de crecimiento neto foliar ( $\mathrm{cm}$ tallo-1 $\left.\mathrm{d}^{-1}\right)$} \\
\hline 14 & & $0.95 \mathrm{Ba}$ & $0.85 \mathrm{Bb}$ & $1.84 \mathrm{Ac}$ & $1.03 \mathrm{Bb}$ & $0.76^{\mathrm{BC}}$ \\
\hline 21 & & $1.04 \mathrm{cb}$ & $1.65 \mathrm{Cab}$ & $3.75^{\mathrm{Ab}}$ & $2.66 \mathrm{Ba}$ & $1.45 \mathrm{cb}$ \\
\hline 28 & & $2.64 \mathrm{Bb}$ & $2.30 \mathrm{Ba}$ & $4.48 \mathrm{Aa}$ & $2.54 \mathrm{Ba}$ & $2.21 \mathrm{Ba}$ \\
\hline & Severo & $1.47 \mathrm{BCa}$ & $1.46 \mathrm{BCa}$ & $3.22 \mathrm{Aa}$ & $2.00 \mathrm{Ba}$ & $1.11 \mathrm{cb}$ \\
\hline & Ligero & $1.61 \mathrm{Ba}$ & $1.75 \mathrm{Ba}$ & $3.49 \mathrm{Aa}$ & $2.14 \mathrm{Ba}$ & $1.84 \mathrm{Ba}$ \\
\hline \multicolumn{2}{|l|}{ EEM } & 0.17 & 0.33 & 0.26 & 0.28 & 0.18 \\
\hline \multicolumn{2}{|c|}{ Frecuencia de pastoreo } & ** & ** & ** & ** & ** \\
\hline \multicolumn{2}{|c|}{ Intensidad de pastoreo } & ns & ns & ns & ns & ** \\
\hline
\end{tabular}

$a b=$ Diferente literal minúscula, en cada columna, indican diferencia $(P<0.05) ; A B C D=$ Diferente literal mayúscula, en cada hilera, indican diferencia $(P<0.05)$; EEM= Error estándar de la media. ns= no significativo; ${ }^{*} P \leq 0.01 ;{ }^{*} P \leq 0.05$. 
senescencia foliar mostraron los mayores valores al pastorear ligeramente la pradera cada 28 días con una densidad menor de tallos.

\section{AGRADECIMIENTOS}

\section{Al proyecto UJAT-PTC-112 del PROMEP por el} apoyo otorgado para la realización de esta investigación.

\section{LITERATURA CITADA}

1 Nantes NN, Euclides VPB, Montagner DB, Lempp B, Barbosa RA, Gois PO. Desempenho animal e características de pastos de capimpiatã submetidos a diferentes intensidades de pastejo. Pesqui Agropecu Bras 2013;48(1):114-121.

2 Ramírez RO, Hernández GA, Carneiro-da Silva S, Pérez PJ , J acaunade Souza JS, Castro RR, et al. Características morfogenéticas y su influencia en el rendimiento del pasto Mombaza, cosechado a diferentes intervalos de corte. Trop Subtrop Agroecosyt 2010;12(2):303-311.

3 Hernández GA, Martínez HP, Mena UM, Pérez PJ, Enriquez QJ . Dinámica del rebrote en pasto insurgente (Brachiaria brizantha Hochst. stapf.) pastoreado a diferente asignación en la estación de lluvia. Téc Pecu Méx 2002; 40: 193-205.

4 Barbosa RA, Junior DD, Euclides VPB, Da silva SC, Zimmer AH, De Almeida RA, et al. Capim-tanzânia submetido a combinações entre intensidade e freqüência de pastejo. Pesqui Agropecu Bras 2007; 42 (3):329-340.

5 Difante GS, Euclides VPB, Nascimento JRD, Da silva SC, Barbosa RA, Torres JR. Desempenho e conversão alimentar de novilhos de corte em capimtanzânia submetido a duas intensidades de pastejo sob lotação rotativa. Rev Bras Zootec 2010;39(1):33-41.

6 Difante GS, Júnior DN, Da Silva SC, Euclides VPB, Montagner DB, Silveira MCT, Pena KD. Características morfogênicas e estruturais do capim-marandu submetido a combinações de alturas e intervalos de corte. Rev Bras Zootec 2011;40(5):955-963.

7 Sbrissia FA, Da silva SC. Compensação tamanho/densidade populacional de perfilhos em pastos de capim-marandu. Rev Bras Zootec 2008;37(1):35-47.
8 Martínez MD, Hernández GA, Enríquez QJF, Pérez PJ, González MSS, Herrera HJG. Producción de forraje y componentes del rendimiento del pasto Brachiaria humidicola ClAT 6133 con diferente manejo de la defoliación. Téc Pecu Méx 2008;46(4):427438.

9 Difante SG, Nascimento JS, Da silva CBV, Euclides MA, Zanine BA. Dinâmica do perfilhamento do capim-marandu cultivado em duas alturas e três intervalos de corte. Rev Bras Zootec 2008;37(2):189196.

10 Inyang U, Vendramini JMB, Sollienberger LE, Sellers B, Adesogan A, Paiva $L$, et al. Forage species and stocking rate effects on animal performance and herbage Responses of 'Mulato' and Bahiagrass pastures. Crop Sci 2010;50:1079-1085.

11 García E. Modificaciones al sistema de clasificación climática de Koppen. 4ạ ed. México: Ed. Limusa; 1988.

12 Palma LDJ, Cisneros DJ. Plan de uso sustentable de los suelos de Tabasco. Fundación Produce Tabasco, A.C. Villahermosa, Tabasco 1996.

13 Hernández-Garay AH, Matthew C, Hodgson J. Effect of spring management on perennial ryegrass and ryegrass-white clover pastures: 2. Tiller and growing point densities and population dynamics. J Agric Res 1997; 40:37-50.

14 Stastistic Analysis System Institute (SAS). Sas User's Guide: Statistics. Version 8. Cary, N.C. Cdroom. 2001.

15 Steel RG, Torrie JH. Bioestadística. Principios y procedimientos. $2^{\text {da }}$ ed. México: McGraw Hill; 1988.

16 Argel PJ, Miles JW, Guiot JD, Lascano CE. Cultivar Mulato (Brachiaria híbrido CIAT 36061): Gramínea de alta producción y calidad forrajera para los trópicos. Cali, Colombia. Centro de Agricultura Tropical (ClAT). 2006.

17 Lara SMA, Pedreira PCG. Respostas morfogênicas e estruturais de dosséis de espécies de Braquiária à intensidade de desfolhação. Pesqui Agropecu Bras 2011;46(7):760-767.

18 Barth NA, De Faccio CPC, Lemaire G, Sbrissia AF, Weber DM, Savian $\mathrm{JV}$, et al. Perfilhamento em pastagens de azavém em sucessão a soja ou milho, sob diferentes métodos e intensidades de pastejo. Pesqui Agropecu Bras 2013;48(3):329-338.

19 Zanine AM, Júnior DN, Sousa BML, Silveira MCT, Silva WL, Santos MER. Tillering dynamics in Guinea grass pastures subjected to management strategies under rotational grazing. Rev Bras Zootec 2013; 42(3): 155-161

20 Sage FR, Kubein SD. The temperature response of C3 and C4 photosynthesis. Plant Cell Envirom 2007;30(9):1086-1106. 